\title{
XRD and UV-Vis Spectroscopic Studies of Lead Tin Sulphide (PbSnS) Thin Films
}

\author{
John Damisa* and Joseph Onyeka Emegha \\ Department of Physics, University of Benin, Benin City, Edo State, Nigeria
}

('Corresponding author's e-mail: john.damisa@uniben.edu)

Received: 9 May 2020, Revised: 1 May 2021, Accepted: 9 May 2021

\begin{abstract}
The effects of deposition cycles on the structural and optical properties of lead tin sulphide ( $\mathrm{PbSnS})$ thin films have been described. Successive ionic layer adsorption and reaction (SILAR) method was used to deposit the ternary material on soda-lime substrates. In the present work, the PbSnS films were grown using lead nitrate, tin chloride dehydrate and thioacetamide solutions as sources of $\mathrm{Pb}, \mathrm{Sn}$ and $\mathrm{S}$, respectively. XRD measurements revealed that the deposited films were polycrystalline in nature with strong adherent to the substrates. The transmittance was found to be high in the near infrared regions of the electromagnetic radiation and, also increased with deposition cycles. The band gap energy was found to vary from 1.70 to $1.75 \mathrm{eV}$ for 10 and 35 deposition cycles. The study indicates that SILAR is an excellent method in depositing good quality films for device applications.
\end{abstract}

Keywords: Thin films, Substrates, Lead tin sulphide, Deposition cycle, Optical properties

\section{Introduction}

Successive ionic layer adsorption and reaction (SILAR) technique is awell-known wet chemical route technique for the deposition of semiconductor thin films. Extensively used for the deposition of oxides, and sulphides, SILAR technique can also be adapted to ternary compounds and doped materials. SILAR has several advantages such as low temperature, low cost of source materials, ease of controlling the deposition parameters, possibility of using any kind of substrates, control of deposition rate and thickness, and convenience for large area depositions [1-3]. Such advantages make SILAR a suitable technique for depositing high quality films in developing countries where facilities for expensive and advanced techniques that are not easy to obtain such as RF-sputtering, molecular beam epitaxy, evaporation, etc. [4].

Tin sulphide $(\mathrm{SnS})$ semiconductor has attracted much attention due to their unique structural, optical and electrical properties. Thin films of $\mathrm{SnS}$ are suitable for various devices such as photo-detectors, infrared detectors, sensing devices and solar cells fabrication [5]. It is an ideal candidate for making costeffective solar cells having exhibited good optical properties, excellent band gap range of 1.3 to $1.5 \mathrm{eV}$, high absorption coefficient $\left(\sim 10^{5} \mathrm{~cm}^{-1}\right)$, good carrier concentrations and hole mobility $\left(0.8-15.3 \mathrm{~cm}^{2} \mathrm{~V}^{-}\right.$ ${ }^{1} \mathrm{~S}^{-1}$ ) [6-8]. Besides these properties, theoretical calculations have indicated a realistic conversion efficiency of above $25 \%$ for SnS-based heterojunction solar cells [6]. However, the maximum efficiency ever reported for SnS-based materials is far below $5 \%$ due to low conductivity [9]. To improve the conductivity of SnS-based heterojunction, several metal dopants have been added to SnS films such as indium, copper, bismuth, silver, antimony, aluminium, iron and lead [9-11].

Bin Hashim [12] studied the effect of aluminum concentration on $\mathrm{SnS}$ thin films by using thermal evaporation method and structural, optical and electrical characterization of the films, while RodriguezCastro et al. [9] studied the SnS thin films that are prepared by ultrasonic spray pyrolysis (USP) technique on corning glass substrates and the effects of copper concentrations on the optical, electrical and structural properties. They found an improvement in the electrical and optical properties of the films with increase in copper incorporations.

In the present study, we report the preparation of ternary lead tin sulphide $(\mathrm{PbSnS})$ thin films by SILAR and physical characterization of the resultant films. The effect of deposition cycles on the structural and optical properties of these films were investigated to find the best condition for the deposition process. 


\section{Materials and methods}

Materials

Commercially available chemicals of analytical grade (AR) were used without further purification for the synthesis of $\mathrm{PbSnS}$ thin films; thioacetamide $\left(\mathrm{C}_{2} \mathrm{H}_{5} \mathrm{NS}\right)$, ammonia solution $\left(\mathrm{NH}_{4} \mathrm{OH}\right)$, triethanolamine $\left(\mathrm{C}_{6} \mathrm{H}_{15} \mathrm{NO}_{3}\right)$ tin chloride dehydrate $\left(\mathrm{SnCl}_{2} \cdot 2 \mathrm{H}_{2} \mathrm{O}\right)$, lead nitrate $\mathrm{Pb}\left(\mathrm{NO}_{3}\right)_{2}$, ethanol $\left(\mathrm{C}_{2} \mathrm{H}_{5} \mathrm{OH}\right)$, acetone $\left(\mathrm{CH}_{3} \mathrm{COCH}_{3}\right)$, and distilled water.

\section{Preparation of crystalline $\mathrm{PbSnS}$ thin films}

In this study, soda-lime substrates with the dimensions of $75 \times 25 \times 1.2 \mathrm{~mm}^{3}$ were used for the deposition. The substrates were washed in a detergent solution, then in acetone, ethanol and distilled water respectively. The washed substrates were dried in an oven at $350 \mathrm{~K}$. For the lead tin sulphide $(\mathrm{PbSnS})$ deposition, the lead nitrate and tin chloride dehydrate were dissolved in $100 \mathrm{~mL}$ of distilled water as the cationic precursors, while thioacetamide dissolved in water was used as the anionic precursor. Triethanolamine (TEA) and ammonia solution were used as stabilizers and $\mathrm{pH}$ adjusters, respectively.

In order to prepare the films, the successive ionic layer absorption and reaction (SILAR) technique was utilized. The deposition process consists of 4 beakers; 2 of which contain the precursors (cation and anion), and the other 2 are filled with distilled water as shown in Figure 1. Each deposition consists of dipping the clean substrates into the solution of the cation (Figure 1(a)), followed by washing in distilled water to detach loosely bound positive ions (Figure 1(b)). The substrates are eventually dipped into the anionic solution to form the required compound (Figure 1(c)), and finally in distilled water (Figure 1(d)). The cycle was repeated 10, 15, 20, 25 and 30 times. The samples were deposited at different cycles to determine the effect of the deposition cycles on the properties of the films. Thereafter, the substrates were dried at $323 \mathrm{~K}$ for $50 \mathrm{~min}$. The samples were later coded as K0 for 10 cycles, K1 for 15 cycles, K2 for 20 cycles, K3 for 25 cycles and K4 for 30 cycles.

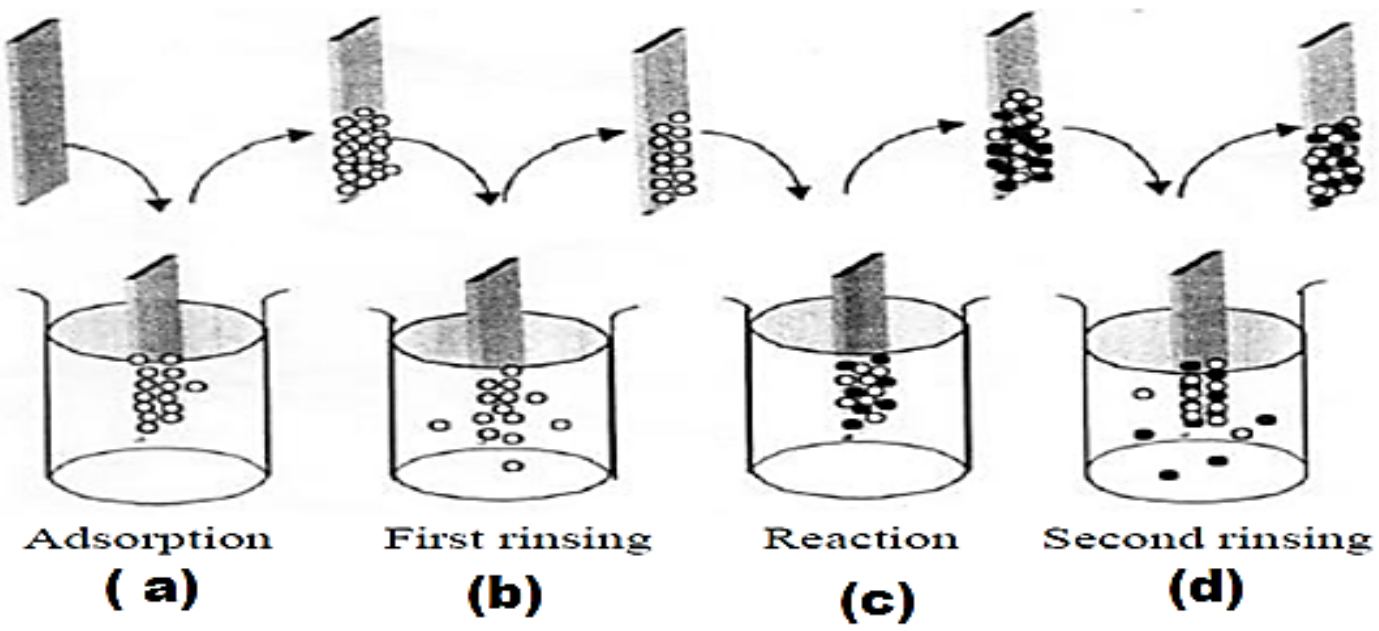

Figure 1 Schematic representation of SILAR method for the deposition of PbSnS thin films: (a) adsorption, (b) first rinsing, (c) reaction and (d) second rinsing of the deposition cycle [13].

\section{Characterization of PbSnSthin films}

The X-ray diffraction (XRD) pattern of the deposited films was measured using Bruker D8 Advanced $\mathrm{X}$-ray diffractometer operating with a $\mathrm{Cu} \mathrm{K \alpha}$ radiation of $1.5406 \AA$. The diffracting angle range was between 15 to $80^{\circ}$ with a scanning rate of $1^{\circ}$ per min.

The UV-Visible optical measurements of PbSnS thin films were done using a UV-1800 Spectrophotometer in a wavelength range of 500 to $900 \mathrm{~nm}$ at room temperature ( $\mathrm{T}=300 \mathrm{~K})$, in a stepsize of 20. The absorption coefficient $(\alpha)$ was estimated using Eq. (1) [14];

$\alpha=\frac{1}{t} \ln \left(\frac{1}{T}\right)$ 
where $t$ is the thickness determines for each of the films. $T$ is the transmittance and it is related to the absorbance (A) by Eq. (2) [15]

$\mathrm{T}=10^{-\mathrm{A}}$

The absorption coefficient $(\alpha)$ is related to the photon energy of the deposited films by the expression in Eq. (3)

$\alpha \mathrm{h} v=\mathrm{k}\left(\mathrm{h} v-\mathrm{E}_{\mathrm{g}}\right)^{\mathrm{n}}$

where $k$ is a constant, $v$ is the frequency, $h$ is the Planks constant, and $\mathrm{E}_{\mathrm{g}}$ is the optical band gap energy. From Eq. (3), the index $n$ is known as the power factor of the electronic transition mode and it is given as $1 / 2$ [16]. The refractive index (n) is given by the relation in Eq. (4) [17]

$\mathrm{n}=\frac{1+\sqrt{R}}{1-\sqrt{R}}$

where $R$ is the reflectance of the films.

\section{Results and discussion}

\section{XRD analysis}

The deposited PbSnS thin films were characterized through XRD measurements in order to determine the crystallinity and lattice parameters of the material. Figure 2 shows the XRD patterns of the films from various deposition cycles. The spectra show diffraction peaks $2 \theta$ at 27.08, 38.76, 52.89 and $65.24^{\circ}$ corresponding to the diffraction lines of (200), (201), (211) and (221). The presence of these diffraction peaks indicate that the deposited films are polycrystalline in nature. It was also observed that the deposited $\mathrm{PbSnS}$ films consist of mixtures of several phases including the orthorhombic $\mathrm{Sn}_{2} \mathrm{~S}_{3}$ (ICDD no 014-0619), $\mathrm{SnS}$ (ICDD no 039-0356) and hexagonal $\mathrm{SnS}_{2}$ (ICDD no 023-0677). Interestingly, it appeared to have a slight distortion in the crystallinity structure of the deposited films on soda-lime substrates as the preferred (111) peak of $\mathrm{SnS}$ at $36^{\circ}$ was absent. Such trend may have resulted from the soda-lime substrates used or the presence of other phases not detected by the XRD. Similar results have been reported in literature $[11,18]$.

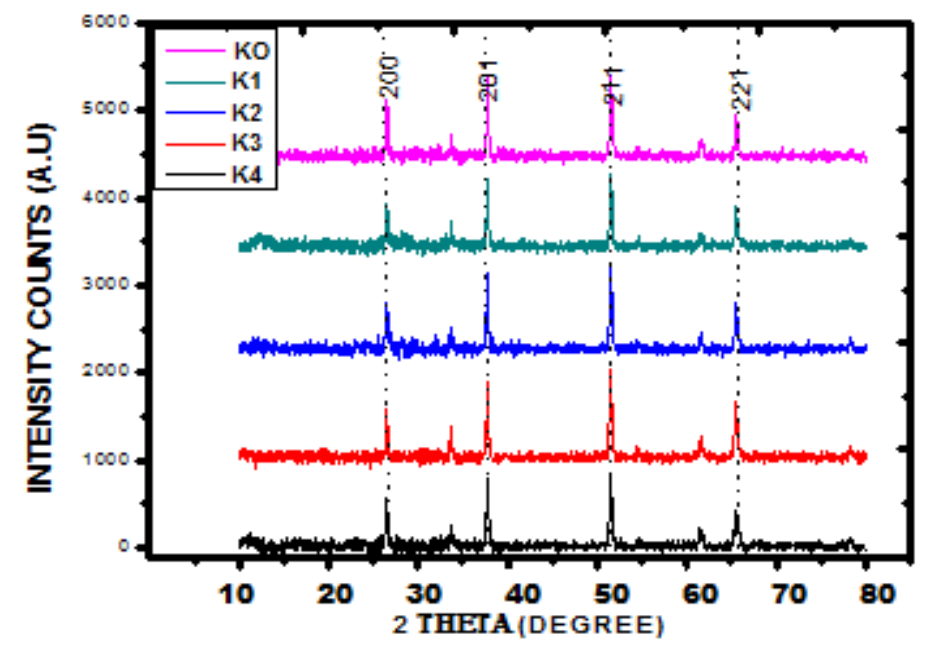

Figure $2 \mathrm{XRD}$ patterns of the deposited $\mathrm{PbSnS}$ thin films.

\section{Optical studies}

In order to have knowledge on the optical properties of the deposited $\mathrm{PbSnS}$ thin films the absorbance, transmittance and reflectance spectra for all the films were measured and some optical parameters such as band gap $\left(\mathrm{E}_{\mathrm{g}}\right)$ and refractive index (n) were analyzed by these spectra. The variation of the absorbance spectra with the incident wavelength is shown in Figure 3. From the figure, it is clearly 
observed that the samples follow similar curve, although differences in their absorbency were observed. Generally, the absorbance depends on the grain sizes, morphology, as well as the film thickness. It was observed from Figure 3 that sample KO (10 cycles), shows a higher optical absorbance than sample K1 (15 cycles) due to the availability of the higher density of the grains in the thin film leading to numerous scattering of the incident light at grain boundaries for absorbing more numbers of photons. However, all films show low absorption on the near infrared region of the electromagnetic spectrum. This consequently indicates an enhancement in the transmission of the films. The nature of the absorption (decreasing with wavelength), of PbSnS thin films makes it a useful material as window layers in solar cells production [19].

The variation of the transmittance spectra with wavelength of the electromagnetic radiation is shown in Figure 4. In all samples, it was observed that the average transmittance was increasing from the visible to the near infrared regions of the wavelength. Also, the average transmittance decreased with increase in the deposition cycles. This may be due to the surface irregularity or defect density within the deposited PbSnS thin films [20]. Similar trend has been reported by Sebastian et al. [10], who assumed that the increased transmittance was a result of the crystallinity and surface topography of the deposited films.

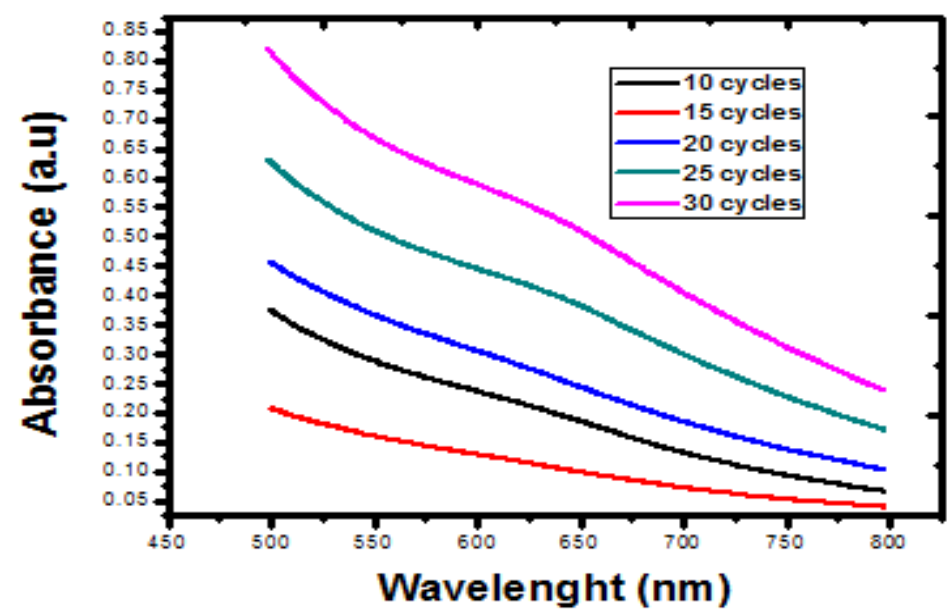

Figure 3Absorbance against wavelength of the deposited $\mathrm{PbSnS}$ thin films.

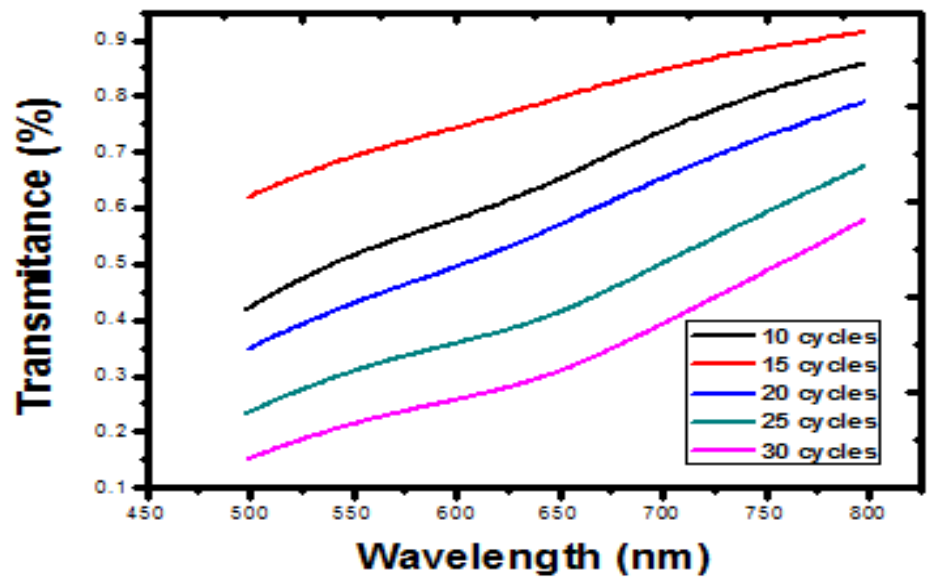

Figure 4 Transmittance of the deposited $\mathrm{PbSnS}$ thin films against wavelength.

Experimentally, the direct band gap energy $\left(E_{g}\right)$ of PbSnS thin films was calculated using the Eq. (3) by plotting $(\alpha h v)^{2}$ against the photon energy (hv). Extrapolating the linear part of the graph to the energy axis at $(\alpha h v)^{2}=0$ gives the band gap energy to be between $1.70-1.75 \mathrm{eV}$, as shown in Table 1 . From Table 1, one can deduce that the band gap increases with deposition cycles, which indicates that the 
band gaps are highly dependent on the deposition cycles. The band gap values showed that $\mathrm{PbSnS}$ is a ternary material whose band gap system falls between the range of the 2 binary constituents of $\mathrm{SnS}$ (1.0$1.47 \mathrm{eV})[6,7]$ and $\mathrm{PbS}(2.1-2.75 \mathrm{eV})[21,22]$. Increasing the deposition cycles shift the bandgap value of the ternary $\mathrm{PbSnS}$ material till it reaches $1.75 \mathrm{eV}$, dueto the gradual replacement of some $\mathrm{Sn}$ atoms by $\mathrm{Pb}$ atoms in the ternary $\mathrm{PbSnS}$ matrix. This trend is probably due to the distributional effects of atoms as a result of the average atomization energy which increases with deposition cycles [23]. Our obtained band gap values are consistent with the work of Sebastian et al.[10] who reported a band gap range of 1.60 to $1.90 \mathrm{eV}$ for $\mathrm{PbSnS}$ thin films by varying the $\mathrm{Pb}$ concentrations.

Table 1 The optical band gap (Eg) and refractive index (n) of PbSnS thin films.

\begin{tabular}{ccc}
\hline Sample codes & Band gaps (eV) & Refractive index (n) \\
\hline K0 & 1.70 & 1.37 \\
K1 & 1.71 & 1.32 \\
K2 & 1.72 & 1.40 \\
K3 & 1.73 & 1.41 \\
K4 & 1.75 & 1.34 \\
\hline
\end{tabular}

The refractive index $(n)$ is an important property of a semiconductor because of its connection with the electronic polarization of ions and the local field within the material [23]. Refractive index is also important in the fabrication of optoelectronic devices such as switches, modulators, filters and so on. The refractive index of $\mathrm{PbSnS}$ films was calculated using the relation in Eq. (4), and it is represented in Figure 5. From the figure, it was observed that the refractive index was increasing with photon energy except for samples K3 (25 cycles) and K4 (30 cycles) that were decreasing at higher photon energies. The average values of the refractive indices rise and fall with deposition cycles as indicated in Table 1. Such behaviors are connected to the successive internal reflection or trapped photon energy in the grain boundaries within the films [14].

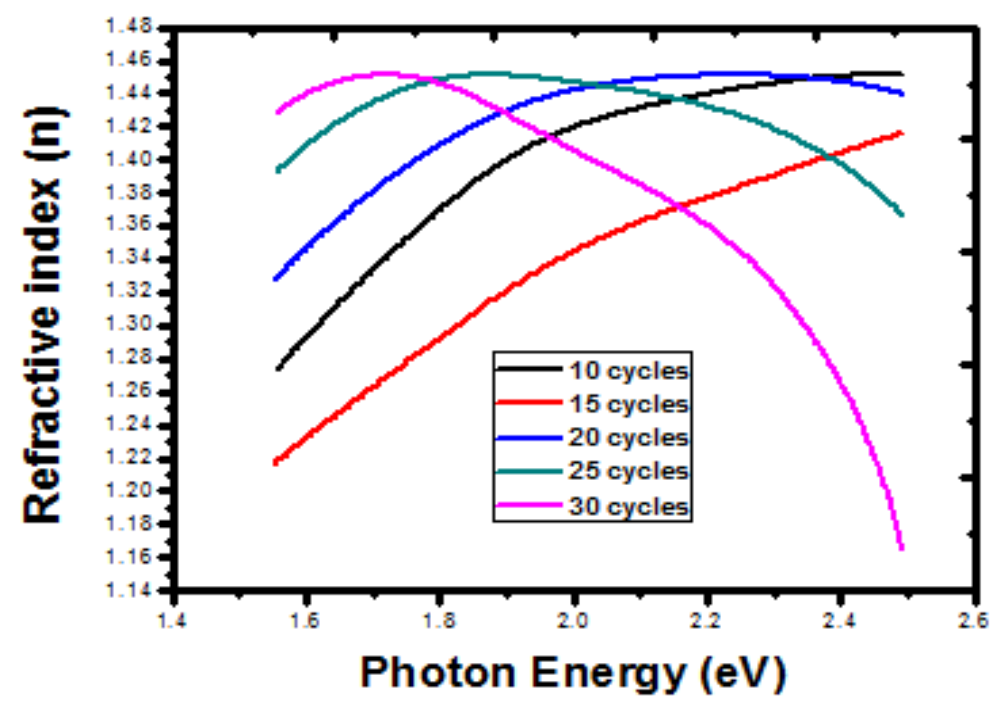

Figure 5 Refractive index against photon energy of $\mathrm{PbSnS}$ thin films. 


\section{Conclusions}

Lead tin sulphide $(\mathrm{PbSnS})$ thin films have been synthesized by SILAR using common chemical reagents at room temperature $(\mathrm{T}=300 \mathrm{k})$. The effect of deposition cycles on the structural and optical properties of the deposited films was investigated using XRD and UV-Vis spectrophotometer. TheXRD measurements revealed changes from the deposited ternary $\mathrm{PbSnS}$ films to binary orthorhombic and hexagonal SnS phases with the preferential (111) crystallographic plane missing. The optical properties showed that the films have an allowed direct transition with band gap energy that varied from 1.70 to 1.75 $\mathrm{eV}$ with increase in deposition cycles. The refractive index was also affected by the deposition cycles as the values varies within the ranges of 1.32 to 1.41 . From this study, it is evident that sample K0 (10 cycles) is the more suitable material for solar cell fabrications.

\section{References}

[1] P Mitra and S Mondal. Structural and morphological characterization of $\mathrm{ZnO}$ thin films synthesized by silar. Progr. Theor. Appl. Phys. 2013; 1, 17-31.

[2] B Ghosh, M Das, P Banerjee and S Das. Fabrication and optical properties of SnS thin films by silar method. Appl. Surf. Sci. 2008; 254, 6436-40.

[3] B Ghosh, S Chowdhury, P Banerjee and S Das. Fabrication of CdS/SnSheterostructured device using successive ionic layer adsorption and reaction deposited SnS. Thin Solid Films 2011; 519, 3368-72.

[4] KG Rao and VK Ashith. Influence of deposition parameters on the structural and optical properties of CdS thin films obtained by micro-controlled silar. J. Phys. Chem. Solid. 2015; 77, 14-22.

[5] AE Oluwalana and PA Ajibade. Effect of temperature and capping agents on structural and optical properties of tin sulphidenanocrystals. J. Nanotechnol. 2019; 2019, 8235816.

[6] SN Nwankwo, S Campbell, RKT Reddy, NS Beattie, V Barrioz and G Zoppi. Temperature controlled properties of submicron thin SnS films. Semicond. Sci. Technol. 2018; 33, 065002.

[7] MO Abou-Helal and M Boshta. Preparation and characterization of tin sulphide thin films by spray pyrolysis technique in ambient temperature. J. Am. Sci. 2012; 8, 61-3.

[8] GH Tariq, K Hutchings, G Asghar, DW Laneand M Anis-Ur-RehmanStudy of annealing effects on the physical properties of evaporated $\mathrm{SnS}$ thin films for photovoltaic applications. J. Ovonic Res. 2014; 10, 247-56.

[9] S Rodriguez-Castro, JN Rios, G Escalante, G Santana, A Sanchez-Juarez and C Alvarez-Macias. Deposition and characterization of $\mathrm{SnS}: \mathrm{Cu}$ thin films by ultrasonic spray pyrolysis technique to applications in photovoltaic devices. In: Proceedings of the IEEE $7^{\text {th }}$ World Conference on Photovoltaic Energy Conversion, Hawaii, USA. 2019, p. 3305-8.

[10] S Sebastian, I Kulandaisamy, S Valanarasu, IS Yahia, HS Kim and D Vikraman. Microstructural and electrical properties evaluation of lead doped tin sulphide thin films. J. Sol-Gel Sci. Technol. 2020; 98, 52-61.

[11] CLC Triana, E Banguero, P Bartolo-Perez and Gordillo. Preparation and characterization of SnS:Bi thin films. Braz. J. Phys. 2011; 41, 15-20.

[12] MFC Hashim. 2017, Morphologies, optical and electrical characterization of aluminum tin sulphide thin films. Master Thesis. Universiti Teknologi Malaysia, Johor, Malaysia.

[13] HM Pathan and CD Lokhande. Deposition of metal chalcogenide thin films by successive ionic layer adsorption and reaction (SILAR) method. Bull. Mater. Sci. 2004; 22, 85-111.

[14] J Damisa, B Olofinjana, O Ebomwonyi, F Bakare, and SO Azi. Morphological and optical study of thin films of $\mathrm{CuAlS}_{2}$ deposited by metal organic chemical vapour deposition technique. Mater. Res. Express 2017; 4, 086412.

[15] JO Emegha, ED Elete, FO Efe and AC Adebisi. Optical and electrical properties of semiconducting $\mathrm{ZnS}$ thin films prepared by chemical bath deposition technique. J. Mater. Sci. Res. Rev. 2019; 4, 18.

[16] JO Emegha, J Damisa, FO Efe, B Olofinjana, MA Eleruja and SO Azi. Preparation and characterization of metal organic chemical vapour deposited copper zinc sulphide thin films using solid source precursors. Eur. J. Mater. Sci. Eng. 2019; 4, 11-22.

[17] FO Efe, B Olofinjana, O Fasakin, MA Eleruja and EOB Ajayi. Compositional, structural, morphological, optical and electrical property evolutions in MOCVD Cu-Zn-S thin films prepared at different temperatures using a single solid source. J. Electron. Mater.2019; 48, 8000-13.

[18] RL Orimi, HK Fadafan and A Asadpour. Effect of Sn concentration on optical and structural properties of $\mathrm{Pb}_{1-\mathrm{x}} \mathrm{Sn}_{\mathrm{x}}$ Snanopowder. Eur. Phys. J. Appl. Phys. 2014; 67, 20404. 
[19] AU Moreh, M Momoh and B Hamza. Influence of substrates temperature on optical properties of nanostructured $\mathrm{CuAlS}_{2}$ thin films grown by two stage vacuum thermal evaporation technique. Int. J. Eng. Sci. Inv. 2013; 2, 48-52.

[20] F Ozutok, K Erturk and V Bilgin. Growth, electrical and optical study of ZnS:Mn thin films. Acta Phys. Pol. A 2012; 121; 221-3.

[21] MM Abbas, AA Shehab, A Al-Samuraee and NA Hassan. Effect of deposition time on the optical characteristics of chemically deposited nanostructure PbS thin films. Energ. Proc. 2011; 6, 241-250.

[22] B Abdallah, A Ismail, H Kashoua and W Zetoun. Effects of deposition time on the morphology, structure and optical properties of $\mathrm{PbS}$ thin films prepared by chemical bath deposition. $J$. Nanomater. 2018; 2018, 1826959.

[23] AS Hassanien and AA Akl. Influence of composition on optical and dispersion parameters of thermally evaporated non-crystalline $\mathrm{Cd}_{50} \mathrm{~S}_{50-\mathrm{x}} \mathrm{Se}_{\mathrm{x}}$. J. Alloy Comp. 2015; 648, 280-90. 